\title{
Unified Propagation Model for Determination of Macrocell GSM (2G, 3G, 4G) Coverage Areas
}

\author{
S. Selim Seker ${ }^{1}$, Nurcin Elif Zeybek ${ }^{2}$ and Suayb Cagri Yener, ${ }^{3,4, *}$ \\ ${ }^{1}$ Department of Electrical-Electronics Engineering, Uskudar University, 34662, Uskudar, Istanbul, Turkey \\ ${ }^{2}$ Department of Electrical-Electronics Engineering, Bogazici University, 34342, Istanbul, Turkey \\ ${ }^{3}$ Department of Electrical-Electronics Engineering, Sakarya University, 54187, Sakarya, Turkey \\ ${ }^{4}$ Electromagnetics Research Center, Sakarya University, 54187, Sakarya, Turkey
}

Received 14 October 2019; Accepted 29 March 2020

\begin{abstract}
Many empirical and theoretical models have been developed, over the past 50 years, to predict coverage areas of propagation. These models are not actually appropriate for every area, since each model was developed in a specific district. These models, no matter how accurate, cause power loss and co-channel interference when they used in the environments where they have not been properly adapted. It's vital to determine the measured field strength for efficient radiocell planning and prediction of the minimum power of transmission required from a base station at a specific frequency to ensure sufficient quality of coverage over a service area. A unified propagation loss model is developed in this work to determine the radio coverage of GSM service areas to improve quality of wireless services for $2 \mathrm{G}, 3 \mathrm{G}$ and $4 \mathrm{G}$. This is the only unified model in the literature. Then, we make site-specific Received Signal Strength measurements and making a detailed comparison of simulation with the measurement results performed at the site.The good agrements are obtain. We also verify our unified propagation loss model with literature.
\end{abstract}

Keywords: Unified model; modelling EM fields, GSM; measurement techniques; cellular systems, wave propagation.

\section{Introduction}

Radio propagation is vital to the technology that emerges for any wireless provider. It is largely site-specific and can vary depending on various features such as operating frequency, antenna properties, etc. Proper characterization of a radio channel by means of a mathematical model is important to estimate signal coverage, available data rates, and the specific performance characteristics of alternative signalization and reception schemes [1]-[5].

Planning is very important before establishing and implementing wireless communication systems. Location propagation characteristics should be known. Generally, duplication provides two types of data corresponding to large-scale path loss of fading problem and small-scale statistics. Knowledge of path loss is very important to know and optimize the coverage of a base station (BS). Without propagation predictions, these parameter estimates can only be obtained with time-consuming and costly electromagnetic field measurements. Propagation path loss (PL) (or attenuation) is the major component in analysis and design of link budget of a communication system [6], [7]. It can be caused by many effects such free space loss, diffraction, refraction, reflection, aperture, medium coupling loss and absorption. Estimation of propagation path loss models has an important role in the design of cellular systems to specify main system parameters such as transmission power, antenna height, frequency etc.

\footnotetext{
*E-mail address: syener@sakarya.edu.tr

Propagation path loss models can be considered into three groups; empirical, deterministic and stochastic [8][16]. Empirical models are basically only based on observations and measurements. While they are computationally efficient, they may not elucidate the specific propagation phenomena [6]. Deterministic models use rules that govern the propagation of electromagnetic waves to determine the strength of a signal received at a particular location. They provide good accuracy but lack in computational efficiency. As onother group, in the case of stochastic models, the environment is modeled by taking into account a number of random variables. Artificial neural networks (ANNs) besed model are also available in the literature claiming that they are more accurate than standard empirical models while being more computationally efficient than deterministic models [15], [17]-[20]

Global System for Mobile Communications (GSM) is in the scope of wireless communication, which depends on the propagation of radio waves in the environment and supplying data transmission [21], [22]. In the early 1990s, the 2nd-generation (2G) cellular systems have been developed [23]. Firstly $3 \mathrm{G}$ and then $4 \mathrm{G}$ systems provide multimedia services and satisfy more requirements in terms of applications and communications. The general trend in the development of wireless communication is the use of higher data rates 5 th-generation $(5 \mathrm{G})$ systems are planned with new technologies and network design [24]-[26]. Since a cellular network depends on the frequency re-use principle, estimated coverage is a very important concept. Hence, to achieve a much more accurate design, the coverage of modern cellular networks and received signal strength 
measurements will be considered as data sources to provide reliable and efficient coverage. Mainly, five different cell sizes in a GSM network, namely macro, micro, pico, femto and umbrella cells can be considered. In this work we will work with macrocells. Macrocell is a cell phone network that ensure the radio coverage provided by a high power (typically tens of watts) base station. Macrocells are generally large, providing a coverage range in kilometers and used for outdoor communication [27]. Generally, they provide coverage larger than microcells.

\section{Developing a Unified Propagation Model}

We have studied the properties of models in detail and in this section, we will develop a unified propagation model which takes the powerful sides of all these propagation models in the literature. Firstly, empirical models were studied. These models are not really appropriate for every area, because each model was developed for a specific district. They have their inputs for different environmental and path parameters and they account for different propagation factors. They were developed from various perspectives and with different original intentions. While all give median transmission loss as an output, some also give location variability and time fading information.

On the other hand, physical models give us more correct results but these models aren't easy-to-implement and needs some complex computations.A unified model will be very helpful in the sense that we could estimate the path loss much more accurately. Now we can compare the propagation models, according to their important parameters such as the distance between transmitter antenna $\mathrm{R}$, and the frequency of the signal $f_{c}$. We will also give some extra variables, which are the valid ranges to apply the model, like the receiver antenna height $h_{\mathrm{r}}$. Table 1 shows below the validity ranges of each model existed in the literature [8][16].

Cellular networks operate on different frequency bands. They are regulated specifically for each country. Mobile frequency band assignments are $900 \mathrm{MHz}$ (for GSM 900), $1800 \mathrm{MHz}$ (for GSM 1800), 2100MHz (for 3G), and $2600 \mathrm{MHz}$ (for $4 \mathrm{G}$ ). We will develop our unified propagation model to these frequency ranges.

First of all, Okumura-Hata is a widely-used model which works well for both urban and suburban areas. For the 900 $\mathrm{MHz}$, we decided to use this model. However, OkumuraHata model is appropriate for the $\mathrm{R}>1 \mathrm{~km}$, as shown in the table above. Therefore, for the distances less than $1 \mathrm{~km}$, we preferred to use the Cost231-Walfisch-Ikegami Model.

Table 1. Validity Ranges of Models

\begin{tabular}{c|c|c|c|c}
\hline Model Name & fc $(\mathbf{M h z})$ & R $(\mathbf{k m})$ & ht (m) & hr (m) \\
\hline Okumura-Hata & $150-$ & $1-10$ & $30-200$ & $1-10$ \\
Cost231-Hata & 1500 & & & \\
& $1500-$ & $1-10$ & $30-200$ & $1-10$ \\
Ibrahim-Parson & $150-$ & $<10$ & $30-300$ & $<3$ \\
Egli & 1000 & & & - \\
Algorithm9999 & $<2000$ & - & - & - \\
Stanford & $>1900$ & $0.1-8$ & $10-80$ & $2-10$ \\
University Interim & & & & \\
(SUI) & $150-$ & $1-20$ & - & - \\
Standard & 3500 & & & $1-3$ \\
Propagation Model & $800-$ & $0.01-5$ & $4-50$ & - \\
Cost231-Walfisch- & 2000 & & & - \\
Ikegami & $300-$ & - & &
\end{tabular}

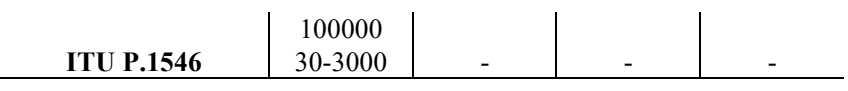

Next, to extend the unified model's validity range to $1800 \mathrm{MHz}$, Cost231-Walfish-Ikegami Model can be utilized as our second model. Please note that, the validity of Okumura-Hata model at this frequency is over. We can also make use of the Cost231-Hata Model since it is a modified version of Hata Model for the frequencies from $1500 \mathrm{MHz}$ to $2000 \mathrm{MHz}$.

Lastly, for the $2100 \mathrm{MHz}$ and $2600 \mathrm{MHz}$ frequencies, our preference is Stanford University Interim Model (SUI), because this model works well for these frequencies and it is valid for both small and large distances between 0.1 and 8 $\mathrm{km}$. Now, in The table 2 given below, we show explicitly which models is used and their corresponding frequency ranges while developing our unified model.

Table 2. Preferred models for the Unified Model

\begin{tabular}{c|c|c|c|c}
\hline $\begin{array}{c}\boldsymbol{R} \\
(\boldsymbol{k m})\end{array}$ & $\boldsymbol{f}_{\boldsymbol{c}}=\mathbf{9 0 0} \mathbf{M h z}$ & $\mathbf{f}_{\mathbf{c}}=\mathbf{1 8 0 0} \mathbf{M h z}$ & $\begin{array}{c}\mathbf{f}_{\mathbf{c}}=\mathbf{2 1 0 0} \\
\mathbf{M h z}\end{array}$ & $\begin{array}{c}\mathbf{f}_{\mathbf{c}}=\mathbf{2 6 0 0} \\
\mathbf{M h z}\end{array}$ \\
\hline $\mathbf{0 . 1 - 1}$ & $\begin{array}{c}\text { Cost231- } \\
\text { Walfisch- }\end{array}$ & $\begin{array}{c}\text { Cost231- } \\
\text { Walfisch- }\end{array}$ & SUI & SUI \\
$\mathbf{1 - 1 0}$ & $\begin{array}{c}\text { Ikegami } \\
\text { Okumura- } \\
\text { Ikegami }\end{array}$ & $\begin{array}{c}\text { Cost231-Hata } \\
\text { Hata }\end{array}$ & SUI & SUI \\
\hline
\end{tabular}

When we consider small frequencies as $900 \mathrm{MHz}$, we could use Ibrahim-Parson, Lee's or Egli's model as well, because their frequency range seems suitable for us. However, they gave many errors as we learned during our simulations. The paper [9] was made for the Benin City of Nigeria. They had estimated path loss by the SUI, Cost231Hata, Lee's and Egli's models, and then plotted against distance on the same graph as that of the measured path loss, which makes a indeed very good comparison of these path loss models for $800 \mathrm{MHz}$. In this paper they had also made Path Loss Model Optimization. In this process the theoretical propagation model is adjusted by considering the measured values provided form the test field data. The objective is to take the estimated field strength as close as possible to the measured field strength. According to research made with $800 \mathrm{MHz}$ frequency, Hata's model gave the closest prediction to the real measurement data with a path loss exponent of 3,40. Based on this closest agreement, Hata's model is selected as the best model for optimization in this research. As can be seen in the [16] Lee's and Egli's models are not suitable to use at all in this frequency. It can be also observed that Stanford University Interim (SUI) will deviate from the real measurement results, as it should be utilized when the frequency is greater than $1900 \mathrm{MHz}$. They didn't use Ibrahim and Parsons Model at all in this research and we will also won't use it, since it was tried in London and it cannot be a general prediction model. So this model cannot be utilized to create our unified model. Therefore, we decided to use Okumura-Hata and Cost231-WalfischIkegami models for $\mathrm{f}_{\mathrm{c}}=900 \mathrm{MHz}$ as they will give much better results.

In the figure 1 below, it can be seen the algorithm which we make use of while developing unified model propagation loss model, in a manner as simple as possible:

Step1.For the frequencies $\mathrm{fc}=900 \mathrm{MHz}$, the unified model will utilize Okumura-Hata Model if the distance from the transmitter to the receiver is between 1 to 10 kilometers. As we mentioned before, Okumura Model is probably the most 
widely quoted of the available models since it is intended for use over a wide variety of radio paths. But several modifications have been developed on Okumura's results. Hata has developed an empirical formula for the propagation loss which is based on Okumura's results [6]. It works very well where the frequency lies in the range $150-1500 \mathrm{MHz}$, base station height lies in the range $30-300 \mathrm{~m}$ and distance lies in the range $1-20 \mathrm{~km}$. It can be found the path loss (L) formula given below:

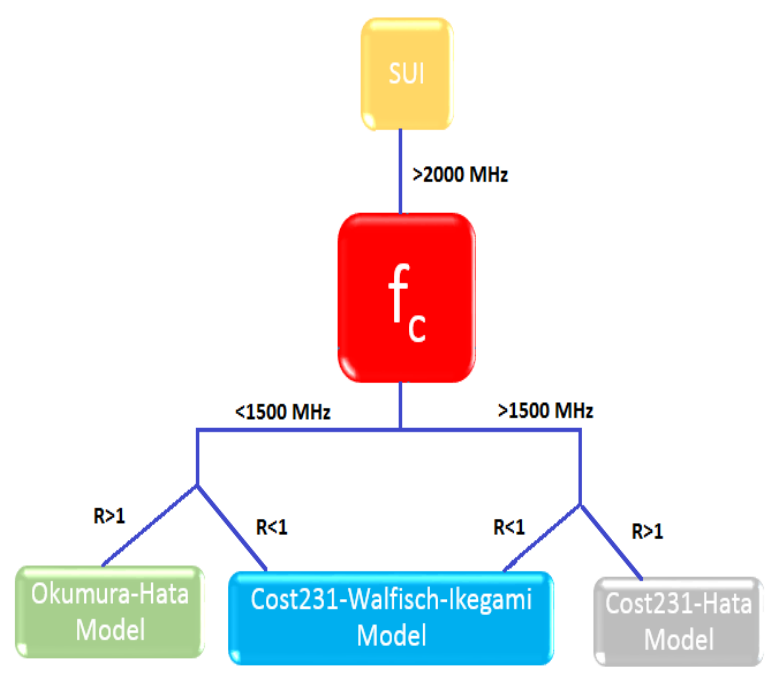

Fig. 1. Algorithm of my Unified Model

$L(d B)=\left\{\begin{array}{cc}A+B+\log d & \text { Urban } \\ A+B+\log d-C & \text { Suburban } \\ A+B+\log d-D & \text { Open }\end{array}\right.$

where A, B, C and D are the parameters used to simplify the (1) and given as:

$$
\begin{aligned}
& A=69.55+26.16 \log f_{c}-13.82 \log \left|h_{b}-a\right| h_{m}|| \\
& B=44.9-6.55 \log \left|h_{b}\right| \\
& C=5.4+2\left[\log \left|f_{c} / 28\right|\right]^{2} \\
& D=40.94+4.78\left[\log \left|f_{c}\right|\right]^{2}-19.33 \log \left|f_{c}\right|
\end{aligned}
$$

where $f_{c}$ is frequency $(150-1500 \mathrm{MHz})$ and $a\left|h_{m}\right|$ is the correction factor for the mobile antenna height.

$$
a\left|h_{m}\right|=\left\{\begin{array}{ccc}
1.1 \log \left|f_{c}\right|-0.7\left|h_{m}\right|-|1.56 \log | f_{c}|-0.8| & \text { Medium or small city } \\
8.28|\log | 1.54 h_{m}||^{2}-1.1 & f_{c} \geq 400 \mathrm{MHz}, \text { large } & \text { city } \\
3.2|\log | 11.25 h_{m} m||^{2}-4.97 & f_{c}<400 \mathrm{MHz}, \text { large } & \text { city }
\end{array}\right.
$$

Hata gives predictions almost indistinguishable from those produced by the Okumura method although Hata's formulations do not include any of the path specific corrections available in the Okumara model. Hata's expressions can be of course very easily implimented to a computer.

Step2.It can be found below the algorithm we will use, if the distance from the Base Transceiver Station is less than 1 kilometer when the frequency is still the same as $900 \mathrm{MHz}$, according to Cost231-Walfisch-Ikegami:

$$
\begin{aligned}
& L[d B]=L F S+L r t s(w r, f, \Delta h M o b i l e, \phi)+L M S D(\Delta h \text { Base, hBase, } d, f, b S) \\
& L F S=32.4+20 \log 10 \cdot d[\mathrm{~km}]+20 \log 10 \cdot f[M H z]
\end{aligned}
$$

Where, LFS: free space path loss, Lrts= roof-to-street loss, LMSD: multi-diffraction loss

$$
\begin{aligned}
& L_{r \mathrm{ss}}=-8.8+10 \log _{10}(f[M H z])+20 \log _{10}\left(\Delta h_{\text {mobile }}[\mathrm{m}]\right)-10 \log _{10}(w[m])+L_{\text {ori }} \\
& L_{\text {ORI }}=\left\{\begin{array}{cc}
-10+0.35 \phi & 0 \leq \phi<35^{\circ} \\
2.5+0.075(\phi-35) & 35^{\circ} \leq \phi<55^{\circ} \\
4-0.114(\phi-55) & 55^{\circ} \leq \phi<90^{\circ}
\end{array}\right. \\
& L_{M S D}=L_{b s h}+k_{a}+k_{d} \log _{10}(d[k m])+k_{f} \log _{10}(f[M H z])-9 \log _{10}(b)
\end{aligned}
$$

Where $\mathrm{L}_{\text {ori }}$ : street orientation function, and

$$
\begin{aligned}
& L_{b s h}=\left\{\begin{array}{cl}
-18 \log _{10}\left(1+\Delta h_{\text {base }}\right) & h_{\text {Base }}>h_{\text {Roof }} \\
0 & h_{\text {Base }} \leq h_{\text {Roof }}
\end{array}\right. \\
& k_{a}=\left\{\begin{array}{cc}
54 & h_{\text {Base }}>h_{\text {Roof }} \\
54-0.8 \Delta h_{\text {Base }} & d \geq 0.5 \mathrm{~km}, h_{\text {Base }} \leq h_{\text {Roof }} \\
54-0.8 \Delta h_{\text {Base }} d[\mathrm{~km}] / 0.5 & d<0.5 \mathrm{~km}, h_{\text {Base }} \leq h_{\text {Roof }}
\end{array}\right. \\
& k_{d}=\left\{\begin{array}{cl}
18 & h_{\text {Base }}>h_{\text {Roof }} \\
18-15 \cdot \Delta h_{\text {Base }} / h_{\text {Roof }} & h_{\text {Base }} \leq h_{\text {Roof }}
\end{array}\right. \\
& k_{f}=-4+\left\{\begin{array}{lc}
0.7(f / 925-1) & \text { medium sized city } \\
1.5(f / 925-1) & \text { metropolitan center }
\end{array}\right.
\end{aligned}
$$

Please note that during simulations, we will take the height data as an array from the terrain profile of each route, originating from the base station.

Step 3. In order to verify if the unified propagation loss model really gives accurate results for the coverage prediction of a city, we will compare our simulation results of the unified model algorithm. For this comparison, actually we will need simulations only in the frequency of $900 \mathrm{MHz}$, because the experimental measurements were done when the mobile frequency band assignment was $900 \mathrm{MHz}$ (for GSM 900).

Step 4. Now, if the frequency is $1800 \mathrm{MHz}$ and provided that the distance is less than $1 \mathrm{~km}$, will utilize Cost231Walfisch-Ikegami formula again as below:

$$
\begin{aligned}
& L=\left\{\begin{array}{cc}
L+L_{r t s}+L_{m s d} & L_{r t s}+L_{m s d}>0 \\
L & L_{r t s}+L_{m s d} \leq 0
\end{array}\right. \\
& L_{r t s}=-16.9-10 \log _{10} w+10 \log _{10} f_{M H z}+20 \log _{10}\left(h-h_{m}\right)+L_{\text {ori }} \\
& L_{\text {ori }}=\left\{\begin{array}{cc}
-10+0.354 \varphi & 0^{\circ} \leq \varphi<35^{\circ} \\
2.5+0.075(\varphi-35) & 35^{\circ} \leq \varphi<55^{\circ} \\
4.0-0.114(\varphi-55) \varphi & 55^{\circ} \leq \varphi<90^{\circ}
\end{array}\right.
\end{aligned}
$$




$$
\begin{aligned}
& L_{m s d}=L_{b s h}+k+k_{d} \log _{10} d_{k m}+k_{f} \log _{10} f_{M H z}-9 \log _{10} b \\
& L_{b s h}=\left\{\begin{array}{cl}
-18 \log _{10}\left[1+\left(h_{b}-h\right)\right] & h_{b}>h \\
0 & h \leq h_{b}
\end{array}\right.
\end{aligned}
$$

$$
\begin{aligned}
& \text { Where } \\
& k_{a}=\left\{\begin{array}{cccc}
54 & & h_{b}>h \\
54-0.8\left(h_{b}-h\right) & h_{b}>h & \text { and } & d_{m} \geq 0.5 \mathrm{~km} \\
54-0.8\left(h_{b}-h\right) \frac{d}{0.5} & h_{b}>h & \text { and } & d_{m}<0.5 \mathrm{~km}
\end{array}\right.
\end{aligned}
$$$$
k_{d}=\left\{\begin{array}{cc}
18 & h_{b}>h \\
18-15 \frac{\left(h_{b}-h\right)}{h} & h_{b} \leq h
\end{array}\right.
$$

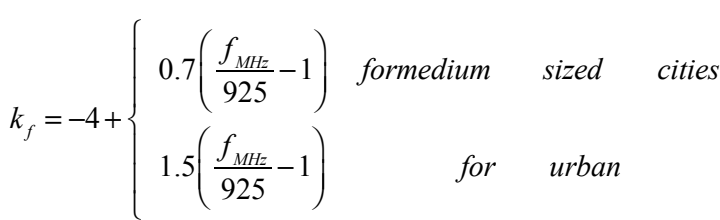

Step 5. Now, if the frequency is $1800 \mathrm{MHz}$ again, but this time the distance is greater than $1 \mathrm{~km}$, we will utilize Cost231-Hata model, shown as below:

$$
L=46.3+33.9 \log f-13.82 \log h_{B}-a\left(h_{R}, f\right)+\left[44.9-6.55 \log h_{B}\right] \log d+C
$$

for sub urban or rural environments:

$$
\begin{aligned}
& a\left(h_{R}, f\right)=(1.1 \log f-0.7) h_{R}-(1.56 \log f-0.8) \\
& C=\left\{\begin{array}{cccccc}
0 & d B & \text { for } & \text { medium cities and suburban areas } \\
3 & d B & \text { for } & \text { metropolitan areas }
\end{array}\right.
\end{aligned}
$$

Step 6. And finally, if the frequency is $2100 \mathrm{MHz}$ or 2600 $\mathrm{MHz}$, whatever the distance is, we will utilize the Stanford University Interim (SUI), given as below:

$\mathrm{La}=\mathrm{A}+10 \mathrm{gamaA} * \log (1000 \mathrm{R} / \mathrm{d} 0)+\mathrm{Xf}+\mathrm{Xhab}+\mathrm{sa}$ $\mathrm{Lb}=\mathrm{A}+10 \mathrm{gamaB} * \log (1000 \mathrm{R} / \mathrm{d} 0)+\mathrm{Xf}+\mathrm{Xhab}+\mathrm{sb}$ $\mathrm{Lc}=\mathrm{A}+10 \mathrm{gamaC} * \log (1000 \mathrm{R} / \mathrm{d} 0)+\mathrm{Xf}+\mathrm{Xhab}+\mathrm{sc}$ $\mathrm{A}=20 * \log (4 * \mathrm{pi} * \mathrm{~d} 0 / \mathrm{lambda})$

lambda $=\mathrm{c} /\left(\mathrm{fc}^{*} 10^{\wedge} 6\right)$

$\mathrm{Xf}=6 * \log (\mathrm{fc} / 2)$

$\mathrm{Xhab}=-10.8 * \log (\mathrm{hm} / 2)$

$\mathrm{Xhc}=-20 \log (\mathrm{hm} / 2)$

gamaA $=4.6-0.0075 \mathrm{hb}+12.6 / \mathrm{hb}$

gamaB $=4-0.0065 \mathrm{hb}+17.1 / \mathrm{hb}$

gamaC $=3.6-0.005 \mathrm{hb}+20 / \mathrm{hb}$

\section{Comparison of Simulation and Measurement Results}

Our experimental site is located in Üsküdar,Istanbul and eight different measurement routes are taken as radials. These radials are chosen at different directions as can be seen in the figure 2 . They include open areas, trees, building covered areas, sea and hilly terrain. The receiving locations along a radial were chosen at about $112 \mathrm{~m}$ apart from each other which is our sampling distance interval used in the calculations. The measurements are made by using some equipment mounted in car in the main streets and by hand held mobile phone in narrow streets and over the sea.

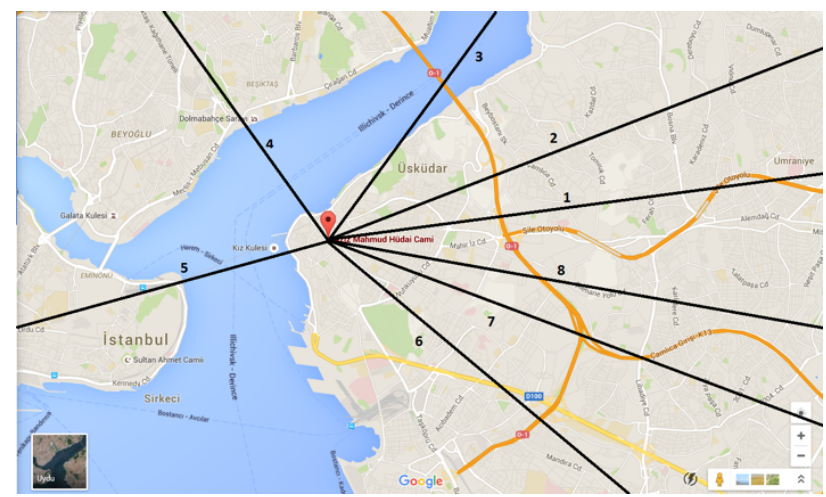

Fig. 2. Eight different measurement routes shown as numbered radials

Measurements are realized by MSTS (Mobile Station Test System) equipment. MSTS is developed for the monitoring of system parameters and the execution of test programs. It is mounted on a car and the transmission between MSTS and the base station is provided by bidirectional antenna. MSTS enables call setup and monitoring of parameters within a GSM network. These parameters are the strength of received radio signal, handover conditions, neighbor cell data, selection of the frequency to be monitored, and suppression of handover by changing of measurement of reports. All parameters can be recorded in files or displayed online on the screen of PC.

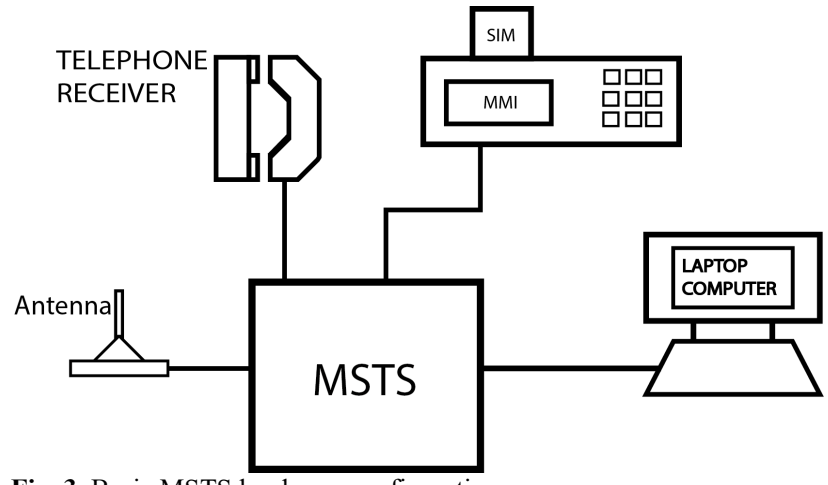

Fig. 3. Basic MSTS hardware configuration

Technical data of the equipment is:

a) Frequency bands: Transmitter $890-915 \mathrm{MHz}$, Receiver 935-960 MHz

b) Carrier spacing: $200 \mathrm{kHz}$

c) Output power: maximum output power $39 \mathrm{dBm}$

d) Receiver characteristics: Sensitivity level -104 $\mathrm{dBm}$, Blocking $-23 \mathrm{dBm}$ for $3 \mathrm{MHz}$ carrier offset

e) Frequency and phase accuracy: Frequency error $0.1 \mathrm{ppm}$ maximum, Phase error 5 degrees rms

f) Power supply: Supply voltage $12 \mathrm{~V}$ DC, Power consumption $150 \mathrm{~W}$

g) Temperature range: 0 to +50 degrees Celsius

On the radials which passes through the main streets, the measurements are realized by the MSTS connected to a Laptop computer mounted in a car. Sector average of received radio signal strength in $\mathrm{dBm}$ is measured continuously. Measurement results are monitored and 
recorded on the computer at the same time. When the car reaches the receiving points on the radials, a numbered mark is put via the keyboard of computer and the mark is displayed on the screen with measured value. Later the marked measurement results are listed for each radial. On the narrow streets and over the sea measurements are realized by Siemens S3 handheld mobile station. The equipment shows the field strengths in $\mathrm{dBm}$ of six different radio signals which it receives all around. Approximate average of the measurement results are taken waiting for five or ten seconds in order to obtain sector averaged or, at least, settled value of the field strength because the equipment shows only the instantaneous values which include the deep notches sometimes due to fading. The measurements on the two routes over the sea are done again by the Siemens mobile station on the ships that transport passengers from Üsküdar to Beşiktaş and to Eminönü which they are located the other side of the bosphorus. The start and stop time of the movement of the ship is recorded and the time difference between these is linearly spaced according to the number of receiving points along the route over the sea. Then the

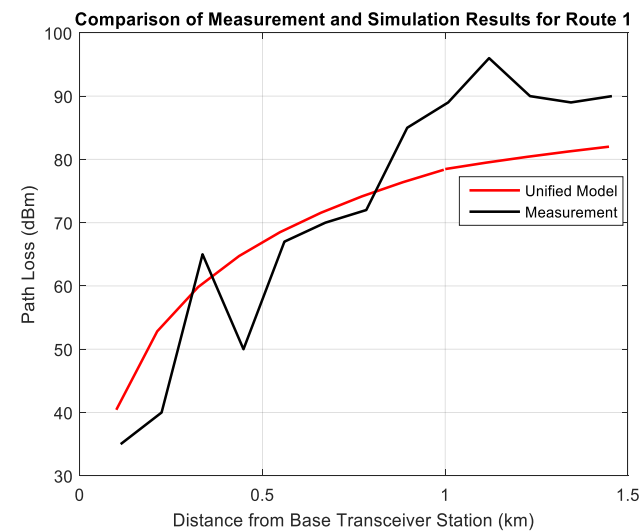

(a)

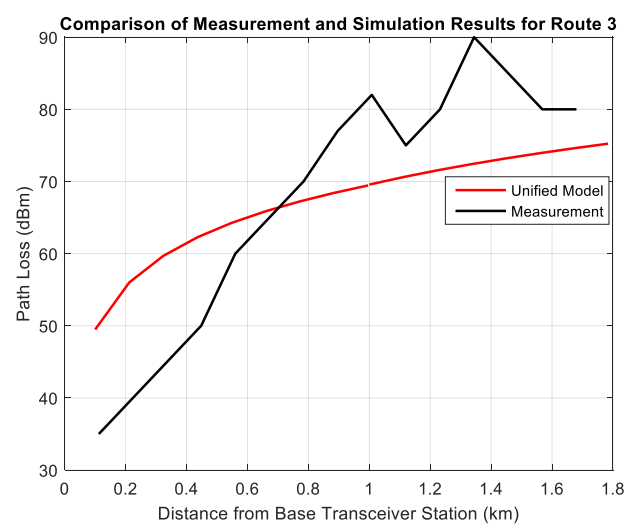

(c)

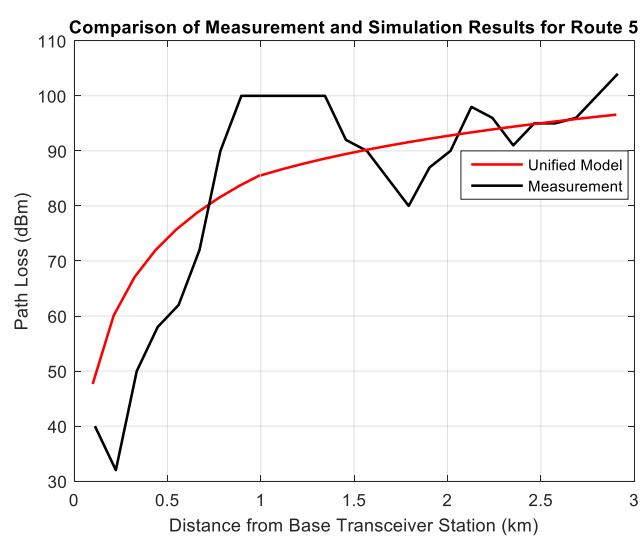

measurement results are obtained at these time intervals in the explained manner above. In the following part, it is seen the plots of experimental measurement results for each route originating from the base station in Üsküdar. Please notice that Route 3, Route 4 and Route 5 are taken over the sea. As can be seen from the figures below, they involve $0 \mathrm{~m}$ height while they go across the Sea of Marmara and the Bosphorus.

These values below are all real data. Also, according to each terrain profile of the all eight routes, we changed the heights accordingly:

hte $=25 \mathrm{~m}$.; Height of the base station antenna from ground in meters

$\mathrm{hm}=1.5 \mathrm{~m}$; Receiver antenna height in meters, $\mathrm{h}=20 \mathrm{~m}$; Average height of the buildings in meters, $\mathrm{w}=10 \mathrm{~m}$; Building separation distance in meters, $\mathrm{fc}=$ $900 \mathrm{MHz}$;Frequency of base station in $\mathrm{MHz}$, fay $=60$; Street orientation angle for the Cost231-Walfisch-Ikegami.The figures $4 \mathrm{a}$-h illustrates simulation of unified model and measurement results for route 1-8.

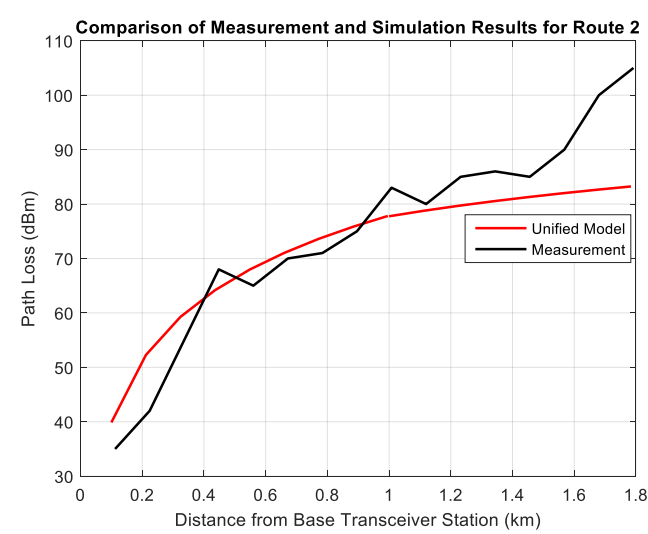

(b)

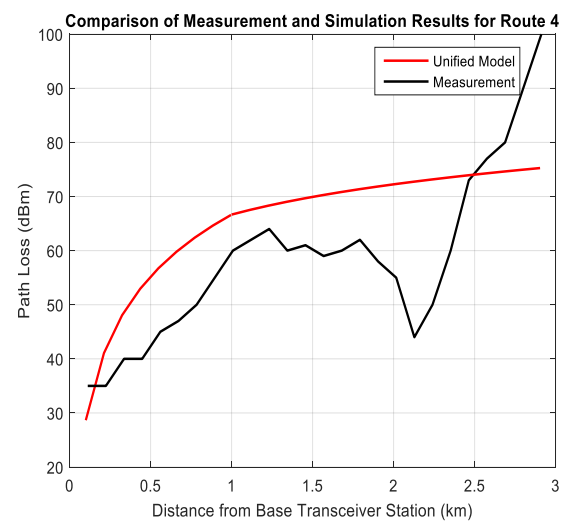

(d)

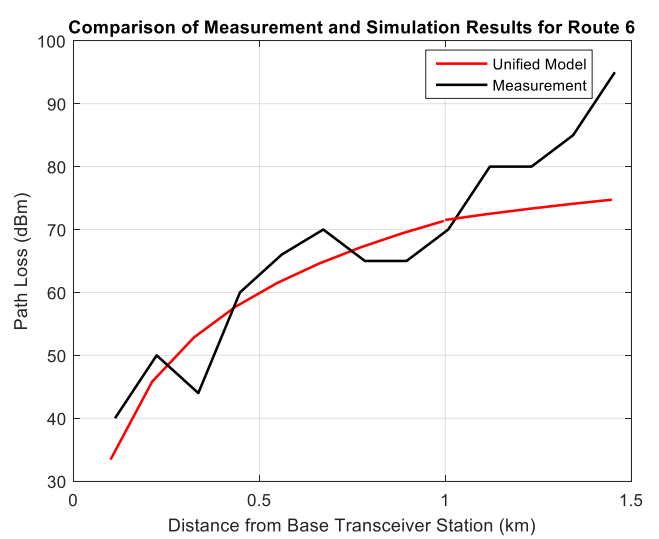


(e)

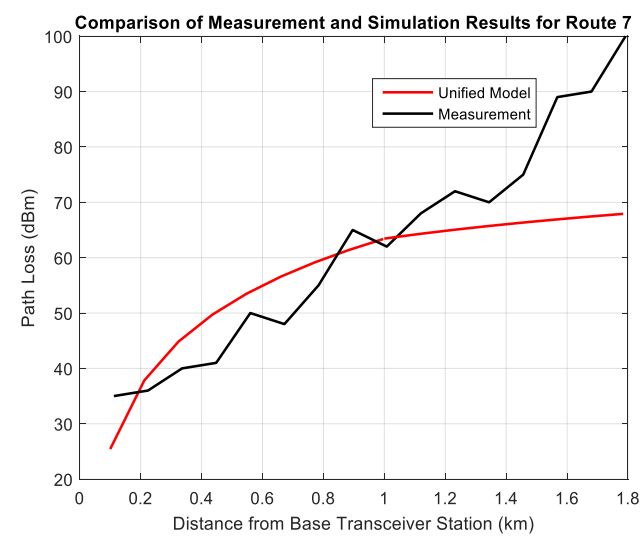

(g) (f)

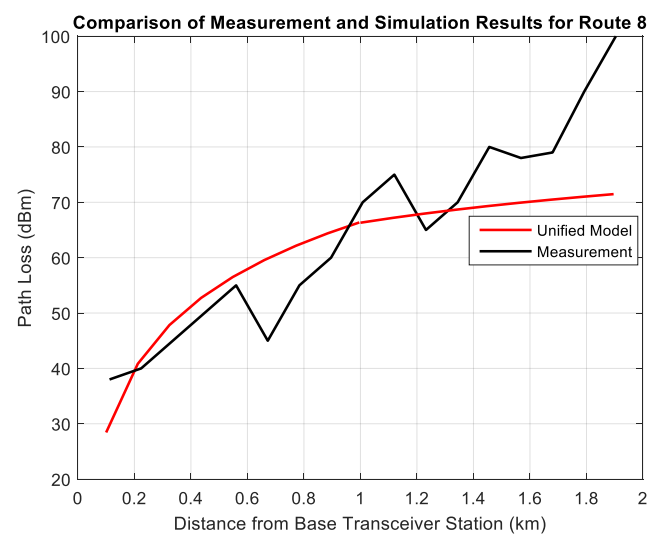

(h)

Fig. 4. (a-h) Simulation results of Unified Model, along with the real measurements results for Route 1-8

We made our simulations of the Unified Model with the frequency of $900 \mathrm{MHz}$. However, in order to make our propagation path loss model more clear, we will also make simulations for the frequencies of $1800 \mathrm{MHz}$ (for2G) ,2100 $\mathrm{MHz}$ (for 3G) and 2600MHz (for4G) using Unified Model. As it can be seen from the plots below, Unified Model gives very accurate information about the propagation path loss in units of $\mathrm{dBm}$. When the distance from Base Transceiver Station is exactly 1 kilometer, the very small gap it can be seen in the plots is due the fact that, we have changed our formula of path loss calculation after the distances become greater than 1 kilometer.

Also, simulation results are indeed very close to the real measurement results. Therefore, we can say that unified propagation path loss model is indeed applicable. Some little deviation in the results may be due to the differences in heights of buildings over each routes, widths of roads, building separation distances and also road orientations with respect to direct radio paths. Also, during the measurements, some miscalibrated equipment may have been used. Please note that, in the routes over the sea, the results deviate less to the routes on the land at further distance. Now let's look at the simulation results of the unified propagation path loss of İstanbul for the frequencies $1800 \mathrm{MHz}$ (Fig.5),2100MHz(Fig.6) and $2600 \mathrm{MHz}$ (Fig.7). We do not have real measurement data for these frequencies, but they are indeed very close to the literature.

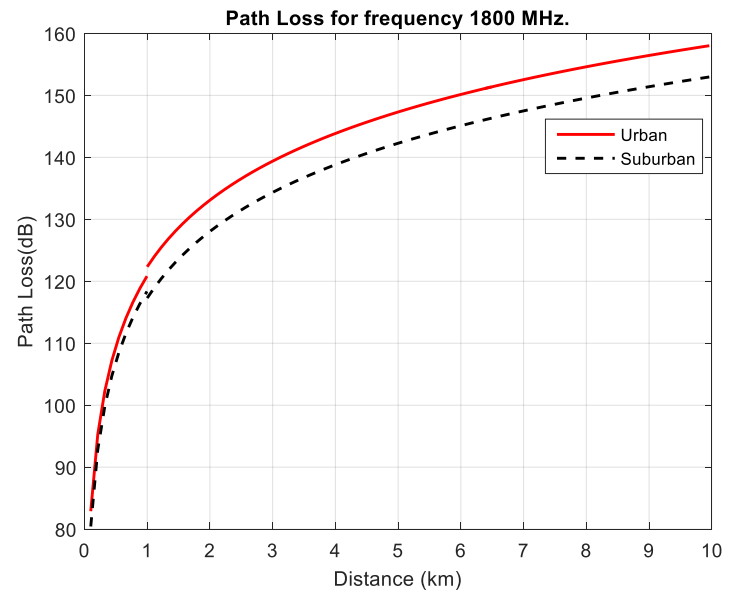

Fig. 5. Simulation results of Unified Model for $\mathrm{f}_{\mathrm{c}}=1800 \mathrm{MHz}$

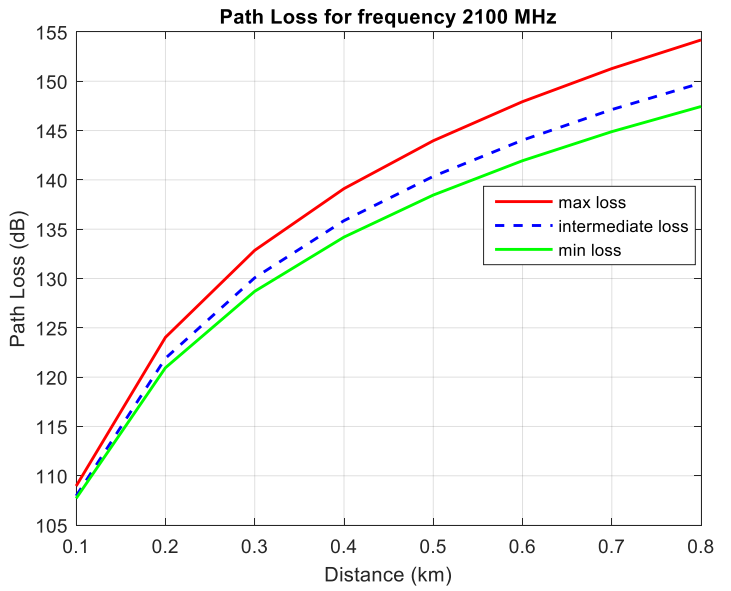

Fig. 6. Simulation results of Unified Model for $\mathrm{fc}=2100 \mathrm{MHz}$

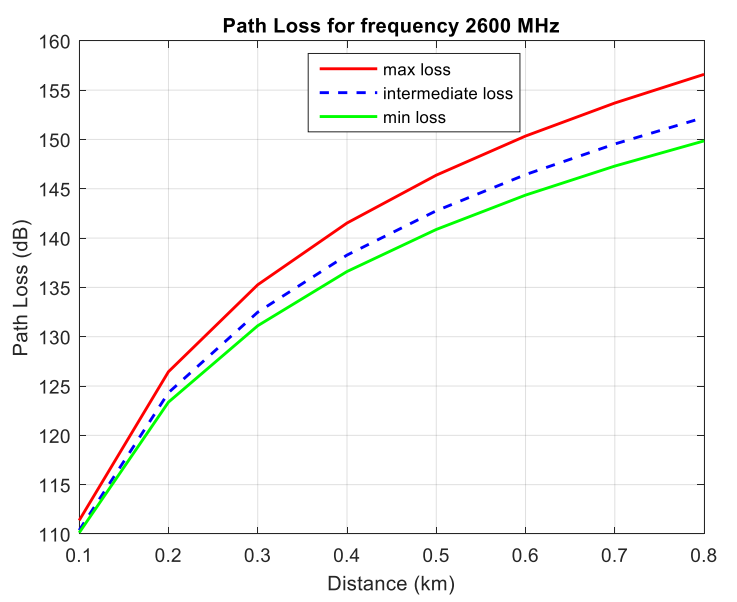

Fig. 7. Simulation results of Unified Model for $\mathrm{fc}=2600 \mathrm{MHz}$

Some of curves are shown are good agreement between model and experiment. There are some differences between model and measurement in some figures. Main reason for his that difficult geological features and terrain profiles of routes and heavy traffic are not allow as to made more experiments.

From the figures 5 and 6 , we can conclude that maximum loss represents hilly terrains, minimum loss on the other hand represents flat terrains. Intermediate loss can be used for the terrain types neither hilly nor flat. We can extent the results of the simulation above, with the building density of the cities. Maximum loss can be used for urban cities with high buildings and dense population. It is proper to use intermediate loss for suburban and min loss for open areas. 
Now we will apply my Unified Model for different frequencies and show the simulation results altogether in the same graph below:

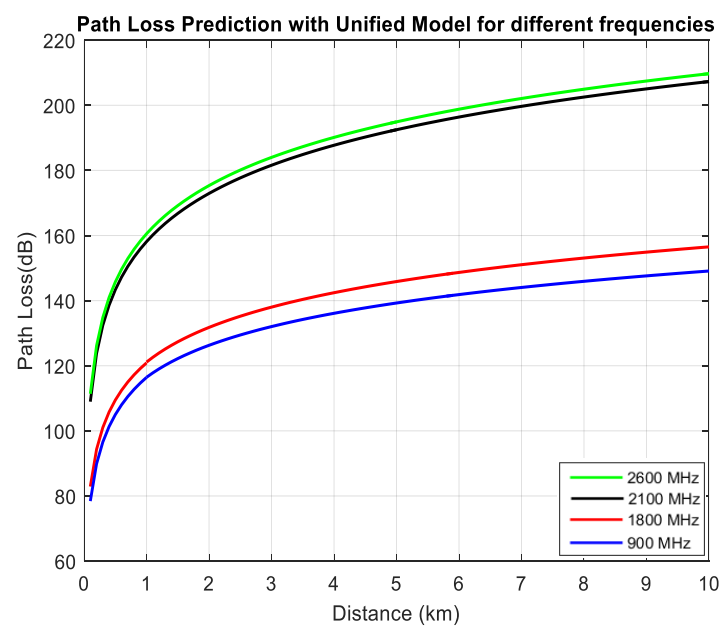

Fig. 8. Application Of Unified Model For Different Frequencies

From figures, if frequency increases from $900 \mathrm{MHz}$ to $2600 \mathrm{MHz}$, attenuation increases as expected, as the frequency increases, the wavelength and power decrease accordingly. Hence, we end up with a lower penetration and a higher path loss.

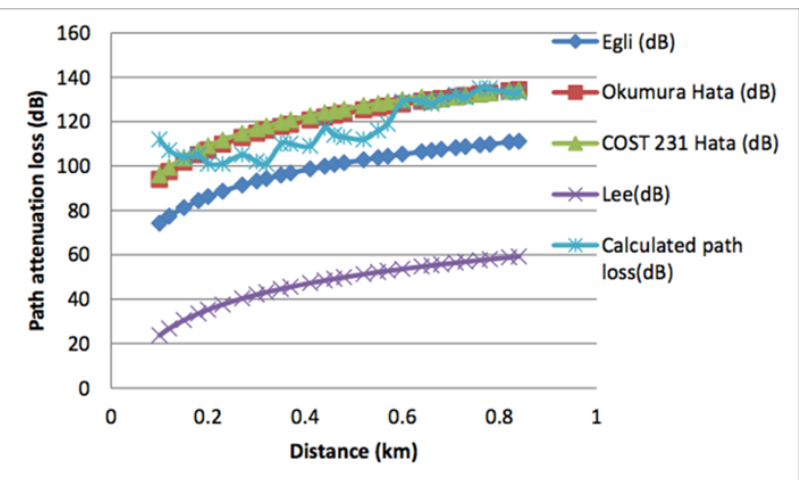

Fig. 9. Path loss prediction at $2100 \mathrm{MHz}$, for the Benin City of Nigeria

The above Figure 9 were taken from ref. [9]. They used a transmitter with height $22 \mathrm{~m}$ and receiver antenna with 1.5 $\mathrm{m}$. Now using their data in the Unified Model,we obtain figure 10.From fig.10,our path loss values are almost the same as the measured path loss. Thus, Unified Model gives us satisfactory results also for the frequencies greater than 2000MHz.

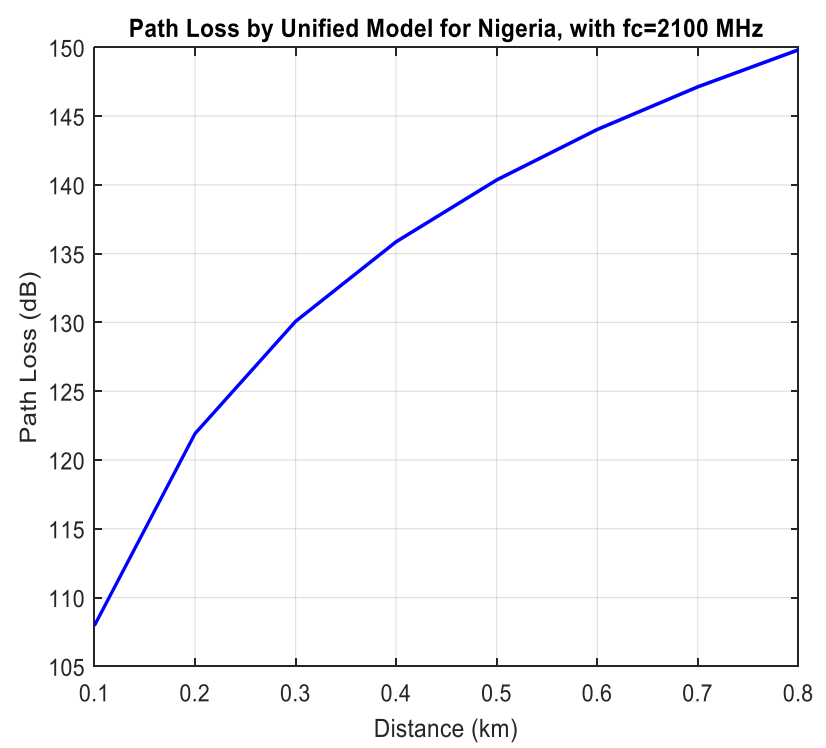

Fig. 10. Path loss simulated by Unified Model for Nigeria at $2100 \mathrm{MHz}$.

\section{Conclusion}

This study will enable us to determine the radio coverage of GSM cells. We have observed that standard empirical path loss models, which are used by many operators worldwide, do not take into consideration the unique geological features and terrain profiles of cities. Therefore, this unique work present an adaptable and suitable unified propagation path loss model. Hence, outcomes of this study will be beneficial as it is both time and cost saving. The unified propagation model gives very close results to the real experimental result,it takes into consideration the unique geological features and terrain profiles of cities, by estimating the loss of signal strength very accurately, it will lead to high level network planning.

\section{Acknowledgments}

The authors would like to thank to Cengiz Riva and Semih Albayrak for their help in realizing this study. Suayb Cagri Yener, would like to express his thanks to Sakarya University Electromagnetic Research Center (SEMAM) for its technical infrastructure during the evaluation of experimental studies.

This is an Open Access article distributed under the terms of the Creative Commons Attribution License

\section{References}

[1] N. G. C, S. U. Ufoaroh, C. O. Ezeagwu, and A. C. Ejiofor, "Path Loss Prediction for Gsm Mobile Networks for Urban Region of Aba , South-East Nigeria," vol. 3, no. 2, pp. 267-281, 2014.

[2] J. B. Andersen and T. S. Rappaport, "Propagation measurements and models for wireless communications channels," IEEE Commun. Mag., vol. 33, no. 1, pp. 42-49, 1995.

[3] Q. Qing He, W. C. Yang, and Y. X. Hu, "Accurate Method to Estimate EM Radiation from a GSM Base Station," Prog. Electromagn. Res. M, vol. 34, pp. 19-27, 2014.

[4] O. Genc, M. Bayrak, and E. Yaldiz, "ANALYSIS OF THE EFFECTS OF GSM BANDS TO THE ELECTROMAGNETIC POLLUTION IN THE RF SPECTRUM," Prog. Electromagn. Res., vol. 101, pp. 17-32, 2010.
[5] M. V. S. N. Prasad, P. K. Dalela, and C. Misra, "EXPERIMENTAL INVESTIGATION OF GSM $900 \mathrm{MHz}$ RESULTS OVER NORTHERN INDIA WITH AWAS ELECTROMAGNETIC CODE AND OTHER PREDICTION MODELS," Prog. Electromagn. Res., vol. 125, pp. 559-581, 2012.

[6] M. Hata, "Empirical formula for propagation loss in land mobile radio services," IEEE Trans. Veh. Technol., vol. 29, no. 3, pp. 317$325,1980$.

[7] A. M. Al-Samman, T. A. Rahman, M. H. Azmi, and M. N. Hindia, "Large-scale path loss models and time dispersion in an outdoor line-of-sight environment for $5 \mathrm{G}$ wireless communications," $A E U$ Int. J. Electron. Commun., vol. 70, no. 11, pp. 1515-1521, Nov. 2016. 
[8] J. Chebil, A. K. Lawas, and M. D. Rafiqul Islam, "Comparison between measured and predicted path loss for mobile communication in Malaysia," World Appl. Sci. J., vol. 21, no. SPECIAL ISSUE1, pp. 123-128, 2013.

[9] I. Joseph and K. C. C, "Urban Area Path loss Propagation Prediction and Optimisation Using Hata Model at $800 \mathrm{MHz}$," IOSR J. Appl. Phys., vol. 3, no. 4, pp. 8-18, 2013.

[10] S. I. Popoola and O. F. Oseni, "Empirical Path Loss Models for GSM Network Deployment in," Int. Ref. J. Eng. Sci., vol. 3, no. 6, pp. 85-94, 2014.

[11]S. S. Seker and G. Apaydin, "New physical discrete UHF multilayer propagation model for urban areas," Appl. Comput. Electromagn. Soc. J., vol. 23, no. 2, pp. 126-133, 2008.

[12]F. Ikegami, S. Yoshida, and T. Takeuchi, "Theoretical prediction of mean field strength for urban mobile radio," IEEE Trans. Antennas Propag., vol. 39, no. 3, p. 4, 1991.

[13]D. Sharma, P. K. Sharma, V. Gupta, R. K. Singh, a S. Path, and L. Model, "A Survey on Path Loss Models Used In Wireless Communication System Design," Engineering, vol. 3, no. 2, pp. 14, 2010.

[14]A. Mousa and M. Najjar, "Optimizing Outdoor Propagation Model based on Measurements for Multiple RF Cell," vol. 60, no. 5, pp. 5-10, 2012.

[15]E. Ostlin, H.-J. Zepernick, and H. Suzuki, "Macrocell path-loss prediction using artificial neural networks," IEEE Trans. Veh. Technol., vol. 59, no. 6, pp. 2735-2747, 2010.

[16]P. M. Ghosh, A. Hossain, A. F. M. Z. Abadin, and K. K. Karmakar, "Comparison Among Different Large Scale Path Loss Models for High Sites in Urban, Suburban and Rural Areas," Int. J. Soft Comput. Eng., vol. 2, no. 2, pp. 287-290, 2012.

[17]A. Nešković, N. Nešković, and D. Paunović, "Indoor electric field level prediction model based on the artificial neural networks," IEEE Commun. Lett., vol. 4, no. 6, pp. 190-192, Jun. 2000.

[18]Z. Stanković, B. Milovanović, M. Veljković, and A. Dordević,
"The hybrid-neural empirical model for the electromagnetic field level prediction in urban environments," in 2004 Seventh Seminar on Neural Network Applications in Electrical Engineering Proceedings, NEUREL 2004, 2004, pp. 189-192.

[19] I. Popescu, D. Nikitopoulos, P. Constantinou, and I. Nafornita, "ANN prediction models for outdoor environment," in IEEE International Symposium on Personal, Indoor and Mobile Radio Communications, PIMRC, 2006.

[20]K. E. Stocker and F. M. Landstorfer, "Empirical prediction of radiowave propagation by neural network simulator," Electron. Lett., vol. 28, no. 8, pp. 724-726, 1992.

[21] V. S. Abhayawardhana, I. J. Wassell, D. Crosby, M. P. Sellars, and M. G. Brown, "Comparison of Empirical Propagation Path Loss Models for Fixed Wireless Access Systems," IEEE 61st Veh. Technol. Conf., vol. 1, no. c, pp. 73-77, 2005.

[22] X.-W. Dai and T. Zhou, "Dual-band reflectarray with crosseddipole elements for GSM and LTE applications," $A E U$ - Int. J. Electron. Commun., vol. 70, no. 5, pp. 605-610, May 2016.

[23] M. F. Iskander and Z. Yun, "Propagation prediction models for wireless communication systems," IEEE Transactions on Microwave Theory and Techniques, vol. 50, no. 3. pp. 662-673, Mar-2002.

[24] T. K. Geok, F. Hossain, and A. T. W. Chiat, "A novel 3D ray launching technique for radio propagation prediction in indoor environments," PLoS One, vol. 13, no. 8, p. e0201905, Aug. 2018.

[25]F. Hossain et al., "An Efficient 3-D Ray Tracing Method: Prediction of Indoor Radio Propagation at $28 \mathrm{GHz}$ in 5G Network," Electronics, vol. 8, no. 3, p. 286, Mar. 2019.

[26] A. Sulyman, A. Nassar, M. Samimi, G. Maccartney, T. Rappaport, and A. Alsanie, "Radio propagation path loss models for 5G cellular networks in the $28 \mathrm{GHZ}$ and $38 \mathrm{GHZ}$ millimeter-wave bands," IEEE Commun. Mag., vol. 52, no. 9, pp. 78-86, Sep. 2014.

[27] S. R. Saunders and A. Aragón-Zavala, Antennas and propagation for wireless communication systems. J. Wiley \& Sons, 2007. 